\title{
Solitary wave solutions of nonlinear evolution and wave equations using a direct method and MACSYMA
}

\author{
Willy Hereman $\dagger$ and Masanori Takaoka $\ddagger$ \\ † Department of Mathematics, Colorado School of Mines, Golden, CO 80401, USA \\ $\ddagger$ Department of Physics, Faculty of Science, Kyoto University, Kyoto, 606, Japan
}

Received 26 March 1990

\begin{abstract}
The direct algebraic method for constructing travelling wave solutions of nonlinear evolution and wave equations has been generalized and systematized. The class of solitary wave solutions is extended to analytic (rather than rational) functions of the real exponential solutions of the linearized equation. Expanding the solution in an infinite series in these real exponentials, an exact solution of the nonlinear PDE is obtained, whenever the series can be summed. Methods for solving the nonlinear recursion relation for the coefficients of the series and for summing the series in closed form are discussed. The algorithm is now suited to solving nonlinear equations by any symbolic manipulation program. This direct method is illustrated by constructing exact solutions of a generalized $\mathrm{KdV}$ equation, the Kuramoto-Sivashinski equation and a generalized Fisher equation.
\end{abstract}

\section{Introduction}

Several methods of obtaining solitary wave solutions have been developed since the inverse scattering technique (IST) was established by Gardner et al (1967). The search for exact solutions of nonlinear PDE outside the range of hyperbolic theory became more and more attractive due to the availability of symbolic manipulation programs. Indeed, MACSYMA, REDUCE, MATHEMATICA, SCRATCHPAD, DERIVE and the like allow one to perform the tedious algebra common to most of the direct methods.

In this paper we are concerned with generating particular solutions of nonlinear evolution and wave equations with constant coefficients by a direct series method. A review of such methods may be found in the paper by Fokas and Ablowitz (1983).

The original idea behind our method goes back to Hirota (1980), who systematically solved large classes of evolution and wave equations by a perturbation approach. Considering the Padé approximant in a power series expansion, Hirota found that it is convenient to transform the original equation into bilinear form for a novel bilinear differential operator. The bilinear equation was then solved by an iteration procedure up to any chosen order, eventually leading to multiple solitary wave solutions $(N$ solitons). Wadati and Sawada (1980) did not require Hirota's preliminary transformations and their 'trace method' allows one to represent the formal series for the $N$-soliton solution in an elegant closed form. However, the above investigators did not pay much attention to the relation between the solution and the exponentials occurring in the expansion.

In 1978, two papers appeared independently, in which solutions of nonlinear evolution equations were constructed from travelling wave solutions of the linear part 
of the equation. In the first paper, Rosales (1978) explored the idea of summing the infinite series either directly or in terms of the inverses of operators in an appropriate space. His aim was to construct multi-soliton solutions by a purely formal iteration procedure starting from all the discrete exponential and continuous (integral) solutions of the linear part of the equation. In the second paper, Korpel (1978) looked at the problem from a more physical point of view. He realized that the final solution of the nonlinear PDE is a rational function of the real exponential solution(s) of the linear part of the equation. Intrigued and motivated by the simple nature of the final solution, Korpel wanted to understand how the nonlinearity mediates the coupling between these real exponential waves.

Once that mechanism of mixing of elementary exponential solutions due to nonlinearity was understood, Hereman et al $(1985,1986)$ established a straightforward algorithm for constructing solitary wave solutions based on Korpel's original idea. This direct algebraic method has since been used to construct solitary wave solutions of coupled systems (Hereman 1988, 1990b, c) and of discrete systems such as the Toda lattice (Dash and Panigraphi 1990). Implicit solutions, e.g. of the Harry Dym equation, have also been constructed by a straightforward generalization of the method (Hereman et al 1989, Banerjee et al 1990). Recently, Coffey (1990a) also generalized the Hereman et al method to recover an analytic closed form solution of a fifth-degree Kdv-like equation and to solve a differential-integral equation exactly.

Seeking travelling wave solutions, one can immediately reduce the nonlinear PDE into a nonlinear ODE and then use basic ideas from the geometrical theory of ODE (Arnold 1983). In particular, Painlevé analysis (Ince 1956) helps in classifying the possible singularities of the solution of the ODE. Partly based on this type of singularity analysis, Takaoka (1989) solved the KdV equation with an extra fifth-order dispersion term. Restricting himself to hyperbolic-type solutions, Takaoka used Mittag-Lefler's theorem and the symmetry of the equation to achieve his goal.

In the direct algebraic method the solution is represented as a series in the real exponential solutions of the linearized equation. The coefficients $a_{n}$ of this series must satisfy a highly nonlinear recursion relation which may be complicated to solve. Furthermore, once the general solution of the recursion relation is obtained, the series has to be summed to arrive at a closed-form solution. The latter step can always be carried out provided the general solution $a_{n}$ of the recursion relation is a polynomial in $n$. As a consequence, the exact solution of the given nonlinear PDE is a rational function of real exponentials.

If Painlevé analysis indicates the existence of analytic (rather than rational) solutions, then it is advantageous to transform the equation immediately into one that admits purely rational solutions. Regarding the solution of the recursion relations, one first determines the degree of the polynomial $a_{n}$ in $n$ and one consequently calculates the unknown coefficients. This straightforward but lengthy calculation is well suited for any symbolic manipulation program such as MACSYMA. The summation of the infinite series is also straightforward, as will become clear in the next section, where we present all the details of the algorithm.

To demonstrate that each step is simple and easy to program, the method is exemplified in sections 3,4 and 5 . We show in section 3 how analytic solutions of a generalized $\mathrm{KdV}(\mathrm{gKdV})$ equation can be obtained. In that section we pay special attention to the transformation of the nonlinear equation. In section 4 , we focus on the solution of the recursion relation for the Kuramoto-Sivashinski (ks) equation. This case is particularly interesting since it is not obvious what the exponential solutions 
of the linear part of the Ks are. Combining the major features of the generalized method, in section 5 we solve the generalized Fisher $(\mathrm{gF})$ equation. Finally, in section 6 we briefly discuss further possible generalizations of the present method, we make some remarks and draw some conclusions.

\section{The improved algorithm}

In this section we discuss the improvements of the algorithm, due to Hereman et al $(1985,1986)$ and Hereman (1988), for the construction of solitary wave solutions of nonlinear PDE. To keep this paper self-contained we will briefly review all the steps of the method. Since the details for the unchanged steps may be found in the key reference by Hereman et al (1986), we will focus on the major improvements in steps $\mathrm{d}$ and $\mathrm{f}$ and in particular in steps $\mathrm{h}$ and $\mathrm{i}$.

Step $a$. Given a nonlinear PDE, with constant coefficients,

$$
\mathscr{F}\left[\partial_{t}, \partial_{x}, u \cdot\right] u=0
$$

where $\mathscr{F}\left[\partial_{t}, \partial_{x}, u\right]$ is a nonlinear operator and $u$ is a function of space $x$ and time $t$, we seek travelling wave solutions. Therefore we introduce a travelling frame of reference defined by the new variable $\xi=x-v t$. The given PDE (1) transforms into a nonlinear ODE for $\phi(\xi) \equiv u(x, t)$. The unknown velocity $v$ of the travelling wave is supposed to be time and space independent. If (1) has a transcendental nonlinearity, e.g. the sine-Gordon equation, then one has to carry out a suitable transformation to remove the transcendental terms (Hereman et al 1986, Hereman 1988).

Step $b$. The resulting ODE is integrated as many times as possible until it becomes an integro-differential equation. Sometimes it is advantageous to multiply the equation by $\phi_{\xi}$ and integrate again. These integrations are not essential and their number varies from example to example. Evolution equations can be integrated at least once; wave equations at least twice.

Step c. In general, the solution $\phi$ may converge to some non-zero constant $C$ as $\xi \rightarrow \pm \infty$, whereas all its derivatives vanish at infinity. For convenience, a change of the dependent variables, according to

$$
\phi=C+\hat{\phi}
$$

assures that the solution $\hat{\phi}$ and its derivatives vanishes at $\xi= \pm \infty$. The constant $C$ will be determined in step $i$. This is in agreement with the series expansion later used in step $g$. Indeed, the exponential solutions of the linear part of the equation also approach zero at $\xi= \pm \infty$. Should it be appropriate, the equation for $\hat{\phi}$ may be integrated again, but without loss of generality, integration constants may now be set equal to zero.

Step $d$. At this point, (Painlevé) singularity analysis may indicate what to do next. A brief discussion of Painlevé analysis may be found in Hereman and Van den Bulck (1988) and Hereman and Angenent (1989), where also a MACSYMA program for the Painlevé test is presented. For our purpose, it suffices to quickly calculate the leading singularity in the solution. Therefore, we substitute $\hat{\phi} \sim \xi^{-p}$ into the equation. Balancing 
the most singular terms determines $p$. If $p$ is not a (positive) integer but a rational number $n_{0} / m_{0}$, then one carries out one more transformation:

$$
\hat{\phi}=\tilde{\phi}^{1 / l_{0} m_{a}} \quad l_{0}=1,2,3, \ldots .
$$

A suitable choice of $l_{0}$ guarantees that the resulting ODE is a polynomial in $\tilde{\phi}$ and all its derivatives. The solution $\tilde{\phi}$ is a rational function in the real exponential solutions of the linearized equation. As a consequence, the coefficients $a_{n}$ in the series expansion in step $\mathrm{h}$ will be of polynomial type in $n$. This will become clear through the examples in sections 3,4 and 5 . Anyway, the equation for $\tilde{\phi}$ remains to be solved.

Step e. Before carrying on one may want to perform the Painleve test for the equation in $\tilde{\phi}$. If the equation passes the test one continues and hopes for a simple exact closed-form solution to exist. If the equation fails the test, one may generalize the search to include implicit solutions (Hereman et al 1989, Banerjee et al 1990), which are out of the scope of this paper.

Step $f$. The main task is to integrate the equation for $\tilde{\phi}$. Motivated by the physics of generation of harmonics and wave mixing, we seek a nonlinear solution built up with decaying real exponentials, $g=\exp (-K(v) \xi)$, which are solutions of the linear part of the equation. If the equation has no linear part, one requires the exponentials to satisfy all the lowest-degree terms. This does not mean that the values for $K(v)$ can always be readily determined from the polynomial $f(K, v)=0$, obtained by substituting $g$ into the linearized equation. This causes no trouble, we just continue with unknown $K(v)$. However, the constraint $f(K, v)=0$ will allow us to partially simplify the recursion relation in the next step. For convenience, one can also use this relation to eliminate $v$ or the highest-degree term in $K(v)$. This often allows one to rescale some coefficients in the nonlinear equation for $\tilde{\phi}$. The example in section 4 will illuminate this procedure.

Step $g$. We expand $\tilde{\phi}$ in a power series

$$
\tilde{\phi}=\sum_{n=1}^{\infty} a_{n} g^{n}
$$

and we substitute this expansion into the full nonlinear equation. Next, we rearrange the sums by using Cauchy's rule for multiple series (Hereman et al 1986), and we equate the coefficient of $g^{n}$, so we obtain a nonlinear recursion relation for the coefficients $a_{n}$. An expansion for the solution like (4) can be motivated as follows: one tries to write the solution of the nonlinear oDE first as a linear approximation and then adds successive nonlinear corrections of higher and higher order to obtain the complete solution.

Step $h$. The actual solution of this recursion relation is carried out in two steps. First, expecting $a_{n}$ to be a polynomial in $n$, we determine its degree. Since $\tilde{\phi}$ behaves like $\xi^{-n_{0} l_{0}}$ (see step d), one may assume $a_{n}$ to have degree $\delta=l_{0} n_{0}-1$. Details on how to accurately calculate the degree $\delta$ are given in (Hereman et al 1986). Hence

$$
a_{n}=\sum_{j=0}^{\delta} A_{j} n^{j}
$$

Replacing $a_{n}$ by (5) in the recursion relation our new task is the calculation of the constant coefficients $A_{j}$. 
Step $i$. The problem is now completely reduced to an algebraic one. The remaining unknowns, being the coefficients $A_{j}$, the values for $K$ and $v$, and possibly $C$ and some integration constants, will follow from solving a nonlinear system. The equations of that system are obtained by setting to zero the coefficients of the different powers of $n$. To do so, we need to calculate the various sums of powers of integers (Spiegel 1968), i.e.

$$
S_{k}=\sum_{i=1}^{n} i^{k} \quad k=0,1,2, \ldots
$$

Formulae for such sums are straightforwardly calculated by hand or easily computed with any symbolic manipulation package. Their expressions also follow from the recursion relation (Spiegel 1968)

$$
(k+1) S_{k}=(n+1)^{k+1}-1-\sum_{i=1}^{k}\left(\begin{array}{c}
k+1 \\
i-1
\end{array}\right) S_{i-1}
$$

with $S_{0}=n$. For example, $S_{1}=(n+1) n / 2, S_{2}=n(n+1)(2 n+1) / 6$, etc. Particularly, the examples in sections 3,4 and 5 will be indicative for this step.

Step $j$. To find the closed-form for $\tilde{\phi}$ we first combine (4) with (5), thus

$$
\tilde{\phi}=\sum_{n=1}^{\infty} \sum_{j=0}^{\delta} A_{j} n^{j} g^{n} \equiv \sum_{j=0}^{\delta} A_{j} F_{j}(g)
$$

with

$$
F_{j}(g) \equiv \sum_{n=1}^{\infty} n^{j} g^{n} \quad j=0,1,2, \ldots .
$$

Considering the relation

$$
F_{j+1}(g)=g F_{j}^{\prime}(g) \quad j=0,1,2, \ldots
$$

one can construct any $F_{j}(g)$ starting from $F_{0}(g)=g /(1-g)$. In particular, $F_{1}(g)=$ $g /(1-g)^{2}, F_{2}(g)=g(1+g) /(1-g)^{3}$, etc.

Step $k$. All that remains to be done is to return to the original variables to obtain the desired travelling wave solution. Retracing the above steps, and inverting the transformations (from $\tilde{\phi}$ to $\phi$ and from $\xi$ to $x$ and $t$ ), the exact closed-form solution of (1) is -at least in principle-obtained.

The process described above may not always work, of course, but it certainly works well for a very large class of very interesting nonlinear evolution and wave equations. Some of these with their solutions are brought together in a large table by Hereman et al (1986).

\section{Example 1: A generalized KdV equation}

\subsection{General treatment}

As an example we consider a gKdV equation,

$$
u_{t}+\left(a+b u^{c}\right) u^{c} u_{x}+d u_{x x x}=0
$$


where $a, b, c$ and $d$ are real constants. This equation contains several interesting and well studied cases dealt with in subsection 3.2. For $c=1$ it is a combined $K d V-m K d V$ equation, which simplifies further to the usual KdV equation provided $b=0$. For $c=2$, (11) was solved explicitly by Dey (1986) using direct integration and later by Coffey (1990a) with the series method. If also $b=0$ then (11) is the $\mathrm{mKdV}$ equation.

Equation (11) with $c=n$ ( $n$ any positive integer) and $b=0$ (or $a=0$ ), is often referred to as the $\mathrm{gKdV}$ equation. This equation describes an anharmonic lattice with a nearest-neighbour interaction force $F \sim \Delta^{n+1}$, where $\Delta$ is the extension or compression of the spring between two neighbouring masses. The $\mathrm{gKdV}$ equation was solved in an early paper by Zabusky (1967), where it was noted that the character and the number of solitary waves depends on whether $n+2$ is even or odd. If $n+2$ is odd there is only one type of solitary wave. If $n+2$ is even one can have a compressive and rarefactive solitary wave. To arrive at these cases, one needs to consider solutions that approach a non-zero constant (background) at infinity. Well-posedness for equations of type (11) (and beyond) has been studied and it is known (Weinstein 1986) that the solitary wave solutions of the $\mathrm{gKdV}$ equation are stable if $n<4$ provided $u(x, 0) \in H^{2}$.

We are interested in the case where $c$ is positive but not necessarily integer and where at least $a$ or $b$ is non-zero. One may expect analytic rather than rational solutions. Dey's and Coffey's investigation showed that (11) admits two kink solutions and two anti-kink solutions $\left((1 \pm \tanh )^{1 / 2}\right.$ form). In passing, Weinstein (1986) also mentions the explicit form of the solitary wave solution for $b=0$ and $c$ integer.

Travelling wave solutions of (11) are of the form $u(x, t)=\phi(\xi)$, where $\xi=x-v t$. The ODE

$$
-v \phi_{\xi}+\left(a+b \phi^{c}\right) \phi^{c} \phi_{\xi}+d \phi_{\xi \xi \xi}=0
$$

can be integrated once to yield

$$
-v \phi+\left(\frac{a}{c+1}+\frac{b}{2 c+1} \phi^{c}\right) \phi^{c+1}+d \phi_{\xi \xi}=C_{1}
$$

where $C_{1}$ is an arbitrary constant. Using $\phi_{\xi}$ as an integrating factor, we get

$-\frac{v}{2} \phi^{2}+\left(\frac{a}{(c+1)(c+2)}+\frac{b}{(2 c+1)(2 c+2)} \phi^{c}\right) \phi^{c+2}+\frac{d}{2} \phi_{\xi}^{2}=C_{1} \phi+C_{2}$

with $C_{2}$ as arbitrary constant.

For simplicity, we continue with $C_{1}=C_{2}=0$, which is equivalent to imposing the boundary conditions $\phi, \phi^{\prime}, \phi^{\prime \prime} \rightarrow 0$ as $\xi \rightarrow \pm \infty$. With reference to step $\mathrm{c}$ in the preceding section, this means that $\tilde{\phi}=\phi$.

To investigate the degree of singularity of $\phi$, we substitute $\phi \sim \xi^{-p}$ into (14) and balance the most singular terms, yielding

$$
\begin{array}{ll}
p=\frac{2}{c} & a \neq 0, b=0 \\
p=\frac{1}{c} & b \neq 0 .
\end{array}
$$

This suggests the transformation

$$
\phi=\tilde{\phi}^{1 / c}
$$


so that (14) may be replaced by

$$
-\frac{v}{2} \tilde{\phi}^{2 / c}+\left(\frac{a}{(c+1)(c+2)}+\frac{b}{(2 c+1)(2 c+2)} \tilde{\phi}\right) \tilde{\phi}^{1+(2 / c)}+\frac{d}{2 c^{2}} \tilde{\phi}^{(2 / c)-2} \tilde{\phi}_{\xi}^{2}=0 \text {. }
$$

Finally, multiplication by $\tilde{\phi}^{2-(2 / c)}$ results in

$$
-\frac{v}{2} \tilde{\phi}^{2}+\left(\frac{a}{(c+1)(c+2)}+\frac{b}{(2 c+1)(2 c+2)} \tilde{\phi}\right) \tilde{\phi}^{3}+\frac{d}{2 c^{2}} \tilde{\phi}_{\xi}^{2}=0
$$

which is a polynomial in $\tilde{\phi}$ and its derivatives.

To get an idea about the nature of the rational solutions of (18), one performs a brief singularity analysis. Substituting $\tilde{\phi} \sim \xi^{-q}$ into (18) and balancing the most singular terms reveals that

$$
\begin{array}{ll}
\tilde{\phi} \sim \xi^{-2} & a \neq 0, b=0 \\
\tilde{\phi} \sim \xi^{-1} & b \neq 0 .
\end{array}
$$

We now substitute the expansion

$$
\tilde{\phi}=\sum_{n=1}^{\infty} a_{n} g^{n}
$$

with $g=\exp (-K(v) \xi)$ into (18). This yields, upon equating powers of $\mathrm{g}$, the nonlinear recursion relation (with $n \geqslant 4$ )

$$
\begin{aligned}
& -v \sum_{l=1}^{n-1} a_{n-l} a_{l}+\frac{2 a}{(c+1)(c+2)} \sum_{l=2}^{n-1} \sum_{m=1}^{l-1} a_{n-l} a_{l-m} a_{m} \\
& +\frac{b}{(2 c+1)(c+1)} \sum_{l=3}^{n-1} \sum_{m=2}^{l-1} \sum_{j=1}^{m-1} a_{n-1} a_{l-m} a_{m-j} a_{j} \\
& +\frac{d K^{2}}{c^{2}} \sum_{l=1}^{n-1}(n-l) l a_{n-l} a_{l}=0 .
\end{aligned}
$$

Herein $K(v)$ is still undetermined. However, note that one readily obtains $K(v)^{2}=$ $c^{2} v / d$ by substituting the exponential $\tilde{\phi}=g=\exp (-K(v) \xi)$ into (18) retaining only the lowest-degree terms. Alternatively, $K(v)$ follows from $(21)$ if we require that $a_{1}$ be arbitrary. Observe that if $a_{n}$ solves $(21)$, as does $a_{0}^{n} a_{n}$ for any $a_{0}$. The knowledge of $K(v)$ or $a_{0}$ is not essential for solving the recursion relation (21).

For $a \neq 0, b=0$, we had $(19 a)$, and from the discussion in step $\mathrm{h}$ it follows that $a_{n}$ will be a polynomial of degree $\delta=1$ in $n$. For $b \neq 0,(19 b)$ indicates that $\delta=0$, thus $a_{n}$ will be constant. Comprising both cases, we take $a_{n}=A_{1} n+A_{0}$. Proceeding as outlined in step $\mathrm{i}$, we substitute $a_{n}$ into (21), and use the appropriate formula (7) for $S_{k},(k=1, \ldots, 5)$ to replace the sums. This leads to a polynomial of degree 6 in $n$, in which we set the different coefficients equal to zero. Finally, one must solve the resulting set of seven coupled nonlinear algebraic equations for the unknowns $A_{0}, A_{1}, v$ and $K(v)$. After a rather tedious calculation, performed by hand or with MACSYMA, one finds the following results.

Case 1: $a \neq 0, b=0$ :

$A_{1}=-\frac{2 v(c+2)(c+1)}{a} \quad A_{0}=0 \quad K^{2}=\frac{c^{2} v}{d} \quad(v$ arbitrary $)$ 
Case 2: $b \neq 0$ :

$$
\begin{aligned}
& A_{1}=0 \quad A_{0}=\frac{a(2 c+1)}{b(c+2)} \\
& v=-\frac{a^{2}(2 c+1)}{b(c+1)(c+2)^{2}} \quad K^{2}=\frac{c^{2} v}{d}=-\frac{a^{2} c^{2}(2 c+1)}{b d(c+1)(c+2)^{2}} .
\end{aligned}
$$

Still treating both cases simultaneously,

$$
\begin{aligned}
\tilde{\phi} & =\sum_{n=1}^{\infty}\left(A_{1} n+A_{0}\right) a_{0} g^{n} \\
& =\left[A_{1} F_{1}\left(a_{0} g\right)+A_{0} F_{0}\left(a_{0} g\right)\right] \\
& =\left[A_{1} \frac{a_{0} g}{\left(1-a_{0} g\right)^{2}}+A_{0} \frac{a_{0} g}{\left(1-a_{0} g\right)}\right] \\
& =-\left[A_{1} \frac{\exp (-K \xi-\Delta)}{(1+\exp (-K \xi-\Delta))^{2}}+A_{0} \frac{\exp (-K \xi-\Delta)}{(1+\exp (-K \xi-\Delta))}\right] \\
& =-\left[\frac{A_{1}}{4} \operatorname{sech}^{2}\left(\frac{K \xi+\Delta}{2}\right)+\frac{A_{0}}{2}\left[1-\tanh \left(\frac{K \xi+\Delta}{2}\right)\right]\right]
\end{aligned}
$$

where we used (9) and were $a_{0}=-\exp (-\Delta)$. The phase constant $\Delta$ follows from the initial conditions if they were specified. Finally, returning to the original variables we obtain the following.

Case 1: $a \neq 0, b=0$ :

$$
u(x, t)=\phi(x-v t)=\left[\frac{v(c+2)(c+1)}{2 a} \operatorname{sech}^{2}\left(\frac{c}{2} \sqrt{\frac{v}{d}}(x-v t)+\frac{\Delta}{2}\right)\right]^{1 / c}
$$

with arbitrary velocity $v$. This formula is in Weinstein (1986).

Case 2: $b \neq 0$ :

$u(x, t)=\phi(x-v t)=\left\{\frac{-a(2 c+1)}{2 b(c+2)}\left[1-\tanh \left(\frac{c}{2} \sqrt{\frac{v}{d}}(x-v t)+\frac{\Delta}{2}\right)\right]\right\}^{1 / c}$

with $v=-a^{2}(2 c+1) /\left[b(c+1)(c+2)^{2}\right]$.

\subsection{Some special cases}

It is easy to recover the well known one-soliton solution of the KdV equation from (25). For instance, for $a=6, b=0, c=d=1$ one gets

$$
u(x, t)=2 k^{2} \operatorname{sech}^{2}\left(k\left(x-4 k^{2} t\right)+\frac{\Delta}{2}\right)
$$

with $k=\sqrt{v / 2}$ and $\Delta$ arbitrary constants. In the derivation of exact solutions one could have carried on with (14) with non-zero integration constants. Indeed, putting $\phi=C+\tilde{\phi}$ then allows one to recover the solution reported in Hereman et al (1985). This is left as an exercise to the reader. The derivation of the sech-solution (Hereman et al 1986) of the $\mathrm{mKdV}$ equation, i.e. (11) with a cubic nonlinearity $u^{2} u_{x}$, is discussed below. 
For $c=1,(11)$ is the combined $\mathrm{KdV}-\mathrm{mKdV}$ equation which has been widely used to model nonlinear phenomena in plasma and solid state physics and in quantum field theory (for references see, e.g., Coffey (1990b)). According to (26), the solution is

$$
u(x, t)=\left(\frac{-a}{2 b}\right)\left[1-\tanh \left(\frac{a}{12} \sqrt{\frac{-6}{b d}}\left(x+\frac{a^{2}}{6 b} t\right)+\frac{\Delta}{2}\right)\right] .
$$

For $c=2$ we retrieve the solution obtained by Dey (1986) and Coffey (1990a) from (26):

$$
u(x, t)=\frac{1}{2} \sqrt{\frac{-5 a}{2 b}}\left[1-\tanh \left(\frac{a}{12} \sqrt{\frac{-15}{b d}}\left(x+\frac{5 a^{2}}{48 b} t\right)+\frac{\Delta}{2}\right)\right] .
$$

Coffey (1990a) also remarks that for $c=1$ the term $u u_{x}$ in (11) can be removed by the substitution $u=w-a / 2 b$. This results in

$$
w_{t}-\left(a^{2} / 4 b\right) w_{x}+b w^{2} w_{x}+d w_{x x x}=0 .
$$

Next, by a trivial change of variables $(t, x) \rightarrow(T, X)$ with $T=t, X=x+\left(a^{2} / 4 b\right) t$, the term in $w_{x}$ in the mKdV equation (30) can be removed. Without any further calculation the exact solution then readily follows from (25) with $c=2$ :

$$
u(x, t)=\sqrt{\frac{6 v}{a}} \operatorname{sech}\left\{\sqrt{\frac{v}{d}}\left[x-\left(v-\frac{a^{2}}{4 b} t\right)\right]+\frac{\Delta}{2}\right\} .
$$

This result is in complete agreement with (2.21) in Coffey (1990a).

Verheest (1988) derived a $\mathrm{gKdV}$ equation

$$
u_{t}+a u^{3} u_{x}+d u_{x x x}=0
$$

for the propagation of ion-acoustic waves at critical densities in a multi-component plasma with different ionic charges and temperatures. By straightforward integration he discovered the solitary wave solution

$$
u(x, t)=\left(\frac{10 v}{a}\right)^{1 / 3} \operatorname{sech}^{2 / 3}\left(\frac{3}{2} \sqrt{\frac{v}{d}}(x-v t)+\frac{\Delta}{2}\right)
$$

with $v$ arbitrary. This solution follows readily from (25) with $b=0, c=3$. It should be obvious now that the nonlinear term in (32) can be of any degree; there will always be analytic solutions similar to (33).

Also in the context of plasma physics, Schamel (1973) derived the equation

$$
u_{i}+u^{1 / 2} u_{x}+d u_{x x x}=0
$$

describing ion-acoustic waves in a cold-ion plasma but where the electrons do not behave isothermally during their passage of the wave. A simple solitary wave solution obtained by Schamel (1973),

$$
u(x, t)=\frac{225 v^{2}}{64} \operatorname{sech}^{4}\left(\frac{1}{4} \sqrt{\frac{v}{d}}(x-v t)+\frac{\Delta}{2}\right)
$$

readily follows from (25). The equation (34) apparently exhibits a stronger nonlinearity than the usual $\mathrm{KdV}$ equation, corresponding to a smaller width and higher velocity of the wave.

Of more interest is the solution of the equation due to Tagare and Chakrabarti (1974)

$$
u_{t}+\left(a+b u^{1 / 2}\right) u^{1 / 2} u_{x}+d u_{x x x}=0
$$


where $u$ refers again to the perturbed ion density in a plasma with non-isothermal electrons, but where a different type of scaling was used than in Schamel (1973). By direct integration, Tagare and Chakrabarti obtained

$$
u(x, t)=\left[\frac{4 a}{15 v}+\frac{\sqrt{75 b v+16 a^{2}}}{15 v} \cosh \left(\frac{1}{2} \sqrt{\frac{v}{d}}(x-v t)+\frac{\Delta}{2}\right)\right]^{-2} .
$$

This solution is valid for all $v$ but

$$
v=-\frac{16 a^{2}}{75 b}
$$

for which (37) would reduce to a constant. One may wonder if there exists a solitary wave solution for the critical velocity (38). The answer is yes, from (26) we have

$$
u(x, t)=\frac{4 a^{2}}{25 b^{2}}\left[1-\tanh \left(\frac{a}{15} \sqrt{\frac{-3}{b d}}\left(x+\frac{16 a^{2}}{75 b} t\right)+\frac{\Delta}{2}\right]^{2}\right.
$$

since $c=\frac{1}{2}$. This particular solution was clearly overlooked in Tagare and Chakrabarti (1974).

This method could also be applied to, e.g., an equation of type (11) but with an extra fifth-order term. Solutions of that type of $\mathrm{gKdV}$ equation were recently reported by Dai and Dai (1989). Our method is also applicable to the generalized Burgers-Huxley equation $u_{1}-\alpha u^{\delta} u_{x}-u_{x x}=\beta u\left(1-u^{\delta}\right)\left(u^{\delta}-\gamma\right)$, for which kink type solutions were obtained very recently by Wang et al (1990).

\section{Example 2: The Kuramoto-Sivashinski equation}

The $\mathrm{ks}$ equation in $1+1$ dimensions

$$
u_{t}+u u_{x}+a u_{x x}+b u_{x x x x}=0
$$

where $a$ and $b$ are real constants, has received a lot of attention, since it serves as a simple model for chaos (Hooper and Grimshaw 1988, Conte and Musette 1989, and the references therein). The ks equation occurs in the context of modelling chemical reaction-diffusion phenomena, flame front instability, propagation of long waves on a thin film or on the interface between two viscous fluids.

The treatment of this example shows some similarity to that of the KdV equation with an additional fifth-order term (Hunter and Vanden-Broeck 1983), $u_{t}+\alpha u u_{x}+$ $\beta u_{x x x}+u_{x x x x x}=0$, for which solitary waves were constructed in Hereman et al (1985, 1986).

We will restrict ourselves to the case $a \neq 0$ and $b \neq 0$, purposely leaving out the Burgers equation $(b=0)$, already solved with our method in Hereman et al (1985).

First introducing a travelling frame of reference, $\xi=x-v t$; subsequently replacing $\phi(\xi) \equiv u(x, t)$ by $C+\tilde{\phi}$ and integrating gives

$$
(C-v) \tilde{\phi}+\frac{1}{2} \tilde{\phi}^{2}+a \tilde{\phi}_{\xi}+b \tilde{\phi}_{\xi \xi \xi}=0
$$

where the integration constant is set to zero right away. Note that we introduced the constant $C$ before the integration; interchanging those two steps causes unnecessary complications. 
For this equation, Painlevé analysis does not indicate the need of an extra nonlinear change of the dependent variable. As a matter of fact, $(41)$ is a polynomial in $\tilde{\phi}$ and its derivatives. However, we learn that $\bar{\phi}$ may have a cubic pole $\left(\tilde{\phi} \sim \xi^{-3}\right)$. Cutting short the steps $d$ and $e$ and proceeding with step $f$ of the algorithm, we solve the linearized equation,

$$
(C-v) \tilde{\psi}+a \tilde{\psi}_{\xi}+b \tilde{\phi}_{\xi \xi \xi}=0
$$

for $\psi=\exp (-K(v) \xi)$, and obtain

$$
f(K, v)=(C-v)-a K-b K^{3}=0 .
$$

Since $v, K(v)$ and also $C$ are unknowns we cannot solve for $K(v)$. We rely on the physical idea behind the direct method to proceed. Indeed, we expect the final solution of the nonlinear equation (41) to be built up from real exponential solutions $g=$ $\exp [-(K(v) / s) \xi]$, where $s$ is (positive) integer. Denoting the roots of (43) by $K_{1}, K_{2}$ and $K_{3}$, these must be integer multiples of a common $\tilde{K}$, i.e. $K_{1}=s_{1} \tilde{K}, K_{2}=s_{2} \tilde{K}$ and $K_{3}=s_{3} \tilde{K}$. This simple ansatz avoids having to work with a triple series for $\tilde{\phi}$ in terms of the exponentials $g_{i}=\exp \left(-K_{i} \xi\right), i=1,2,3$. For more details on this crucial step see Hereman et al (1986).

Motivated by the above arguments, let us seek a solution of the form

$$
\tilde{\phi}=\sum_{n=1}^{\infty} a_{n} g^{n}
$$

Substitution of this expansion into (41) yields the recursion relation

$$
\left[(C-v)-\frac{n}{s} K a-\left(\frac{n}{s}\right)^{3} b K^{3}\right] a_{n}+\frac{1}{2} \sum_{i=1}^{n-1} a_{l} a_{n-1}=0 \quad n \geqslant 2
$$

with $a_{1}$ arbitrary if $s=1$ and $a_{1}=0$ if $s \neq 1$. From (43) we can isolate either $C-v$ or $b K^{3}$ to simplify (45). In order to avoid cubic terms in $K$ we opted for the latter, and replace $(45)$ by

$2\left(\frac{n}{s}-1\right)\left\{\left[\left(\frac{n}{s}\right)^{2}+(v-C)\left(\frac{n}{s}\right)+1\right]+\frac{a K n}{s}\left(\frac{n}{s}+1\right)\right\} a_{n}+\sum_{l=1}^{n-1} a_{l} a_{n-l}=0$.

Next, we calculate the degree $\delta$ of $a_{n}$ in $n$. For $\tilde{\phi}$ may have a cubic pole, $\delta=2$. Alternatively, one could quickly substitute $a_{n} \sim n^{\delta}$ into (45) and balance the highestdegree terms in $n$; the catch is that every sum increases the degree of $n$ by one. Doing so, from $\delta+3=2 \delta+1$ it follows that $\delta=2$. Substituting

$$
a_{n}=A_{2} n^{2}+A_{1} n+A_{0}
$$

into (46), then applying the formulae for $S_{0}$ through $S_{4}$, as given in (7), will eventually give rise to a polynomial of degree 5 in $n$. The six coefficients of this polynomial are set equal to zero, yielding

$$
\begin{aligned}
& A_{0}\left[2(C-v)-A_{0}\right]=0 \\
& 60 A_{1} s(C-v)-A_{2}^{2} s+10 A_{0} A_{2} s-5 A_{1}^{2} s-30 A_{0} A_{1} s+30 A_{0}^{2} s-60 a A_{0} K=0 \\
& 12 A_{2} s(C-v)-A_{1} A_{2} s-6 A_{0} A_{2} s+6 A_{0} A_{1} s-12 a A_{1} K=0 \\
& 12 A_{0}(C-v)-4 A_{0} A_{2} s^{3}-A_{1}^{2} s^{3}+12 a A_{2} K s^{2}-12 a A_{0} K=0 \\
& A_{1}\left[12(C-v)-A_{2} s^{3}-12 a K\right]=0 \\
& A_{2}\left[60(C-v)-A_{2} s^{3}-60 a K\right]=0 .
\end{aligned}
$$


This nonlinear system for the unknowns $A_{0}, A_{1}, A_{2}, K, C-v$ and the integers $s$ is readily solved. The condition (43) determines $b$ as soon as $K$ and $C-v$ are found. The solution as we obtained it with MACSYMA, is summarized in table 1 .

What remains to be done is to write $\tilde{\phi}$ in its closed form. Using (9) and (10), we have

$$
\begin{aligned}
\tilde{\phi} & =\sum_{n=1}^{\infty}\left(A_{2} n^{2}+A_{0}\right)\left(a_{0} g\right)^{n} \\
& =A_{2} F_{2}\left(a_{0} g\right)+A_{0} F_{0}\left(a_{0} g\right) \\
& =A_{2} \frac{g\left(1+a_{0} g\right)}{\left(1-a_{0} g\right)^{3}}+A_{0} \frac{a_{0} g}{\left(1-a_{0} g\right)} \\
& =\frac{1}{4} A_{2} \frac{\left(1+a_{0} g\right)^{3}}{\left(1-a_{0} g\right)^{3}}+\frac{1}{4}\left(2 A_{0}-A_{2}\right) \frac{\left(1+a_{0} g\right)}{\left(1-a_{0} g\right)}-\frac{1}{2} A_{0} .
\end{aligned}
$$

If we select the constant $a_{0}=-\exp (-\Delta)$ to be negative and if we absorb it into the exponential $g$ as an arbitrary phase $\Delta$, then

$\tilde{\phi}=\frac{1}{4} A_{2} \tanh ^{3}\left(\frac{(K / s) \xi+\Delta}{2}\right)+\frac{1}{4}\left(2 A_{0}-A_{2}\right) \tanh \left(\frac{(K / s) \xi+\Delta}{2}\right)-\frac{1}{2} A_{0}$.

This form still covers all the cases for $s$. We now return to the original variables $\phi=C+\tilde{\phi}$ and list the remaining cases.

Case 1: $s=1$ :

$$
\phi=v+\frac{15 a K}{19}\left[11 \tanh ^{3}\left(\frac{K \xi+\Delta}{2}\right)-9 \tanh \left(\frac{K \xi+\Delta}{2}\right)\right]
$$

where $K=\sqrt{11 a / 19 b}$.

Case 2: $s=2,3$ or -5 :

$$
\phi=v-\frac{15 a \tilde{K}}{19}\left[\tanh ^{3}\left(\frac{\tilde{K} \xi+\Delta}{2}\right)-3 \tanh \left(\frac{\tilde{K} \xi+\Delta}{2}\right)\right]
$$

with $\tilde{K}=\sqrt{-a / 19 b}$.

In both cases the velocity $v$ is completely free, confirming that the KS equation is invariant under the Galilean transformation (Conte and Musette 1989). Of course, $u(x, t)$ is obtained by replacing $\xi$ by $x-v t$ in (51) and (52).

Hooper and Grimshaw (1988) report the solution (51), as shown in figure 1 (for $v=\Delta=0, K=2$ and $\left.a=\frac{19}{30}\right)$ but were clearly not aware of the solution (52) shown in

Table 1. Solutions of the algebraic equations for the kS case.

\begin{tabular}{lllll}
\hline Unknowns & $s=1$ & $s=2$ & $s=3$ & $s=-5$ \\
\hline$A_{0}$ & $60 a K / 19$ & $30 a K / 19$ & $20 a K / 19$ & $-12 a K / 19$ \\
$A_{1}$ & 0 & 0 & 0 & 0 \\
$A_{2}$ & $660 a K / 19$ & $-30 a K / 19$ & $-20 a K / 19$ & $12 a K / 19$ \\
$C-v$ & $30 a K / 19$ & $15 a K / 19$ & $10 a K / 19$ & $-6 a K / 19$ \\
$b$ & $11 a /\left(19 K^{2}\right)$ & $-4 a /\left(19 K^{2}\right)$ & $-9 a /\left(19 K^{2}\right)$ & $-25 a /\left(19 K^{2}\right)$ \\
\hline
\end{tabular}




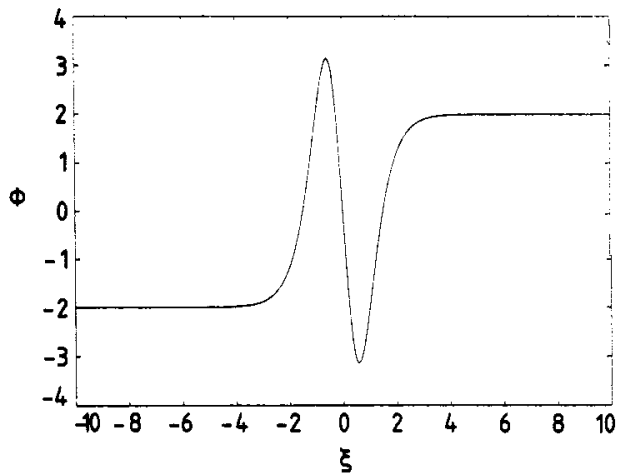

Figure 1. The shock profile solution $\phi=$ $11 \tanh ^{3}(\xi)-9 \tanh (\xi)$ of the $\mathrm{ks}$ equation.

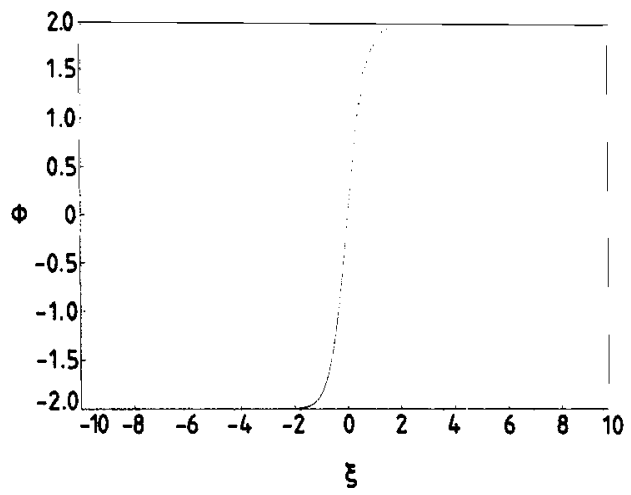

Figure 2. The kink-type solution $\phi=3 \tanh (\xi)-$ $\tanh ^{3}(\xi)$ of the $\mathrm{ks}$ equation.

figure $2\left(v=\Delta=0, a=\frac{19}{30}\right.$ and $\left.\tilde{K}=2\right)$. Although both have the same type of formula they differ drastically in shape. The exact solution (51) assumes the familiar (kink-type) shock profile, similar to the one for the Burgers equation (40) with $b=0$ whereas (52) is a special shock profile. Both kink-type solutions can also be obtained from PainlevéBäcklund equations (Conte and Musette 1989). Singular kink-type solutions could be obtained by taking $a_{0}>0$. Allowing complex values for $K$, to be precise purely imaginary values, would lead to periodic but singular solutions (built up with $\tan$ and $\tan ^{3}$ ).

It is worthwhile to return to the recursion relation (45). For the first case $(s=1)$, the roots of (43) are $K_{1}=-K_{2}=\sqrt{11 a / 19 b}$ and $K_{3}=0$, and $C=v+30 a K_{1} / 19$. Hence, the coefficient of $a_{n}$ in (45) factors into

$$
-(a K / 19)(n-1)\left(11 n^{2}+11 n+30\right) \text {. }
$$

This implies that $a_{1}$ can be taken arbitrary and different from zero.

For the second case $(s=2,3,-5)$, the roots of (43) are $K_{1}=2 \tilde{K}, K_{2}=3 \tilde{K}, K_{3}=-5 \tilde{K}$, with $\tilde{K}=\frac{K}{s}=\sqrt{-a / 19 b}$. Obviously, $a_{1}=0$, and the coefficient of $a_{n}$ in (45), i.e.

$$
30 a \tilde{K} / 19-a n \tilde{K}-b n^{3} \tilde{K}^{3}
$$

factors into

$$
(a \tilde{K} / 19)(n-2)(n-3)(n+5) .
$$

This implies that $a_{2}$ and $a_{3}$ can be taken arbitrary. In retrospect, should we have required that (54) was zero for $n=2$ and $n=3$ we would have found $\hat{K}=\sqrt{-a / 19 b}$ and $C-v=30 a \tilde{K} / 19$ right away. Other selections for the values of $n$ lead to the same result after relabelling the $a_{n}$. This less rigorous procedure for determining the wavenumber $\tilde{K}$ and wave velocity $v$ was used in Hereman et al $(1985,1986)$ to solve the $\mathrm{KdV}$ equation with an extra fifth-order dispersive term. The present modifications of the method make this step much more solid and applicable without hindsight.

\section{Example 3: The generalized Fisher equation}

\subsection{General treatment}

The Fisher equation,

$$
u_{t}-u_{x x}-u(1-u)=0
$$


plays an important role in biophysics, where it describes the propagation of mutant genes. This nonlinear diffusion equation also describes the spread of flames and explosions, the evolution of a neutron population in a nuclear reactor and the branching Brownian motion process. The Fisher equation was solved by Ablowitz and Zeppetella (1979) with a series solution method similar to the one presented in this paper.

Obviously, one may wonder what happens if the quadratic nonlinearity in the Fisher equation were replaced by a cubic or higher-degree one. Using direct integration, a solitary wave solution of the $\mathrm{gF}$ equation,

$$
u_{\mathrm{t}}-u_{x x}-u\left(1-u^{c}\right)=0
$$

with $c$ a real constant, was found by Wang (1988).

Equation (57) reduces to the real Newell-Whitehead ( $N w$ ) equation for $c=2$, which itself was recently solved by Cariello and Tabor (1989). The solution of the latter may also be found in Hereman (1990a) on the FitzHugh-Nagumo (FHN) equation. By formally setting $a=-1$ in the celebrated $\mathrm{FHN}, u_{t}-u_{x x}+u(1-u)(a-u)=0$, the real NW equation is found. Their solutions are related in a similar way.

We apply the direct method to (57), where $c$ does not have to be integer. We expect (57) to have analytic rather than rational solutions expressible in real exponentials.

Considering only travelling wave solutions that will vanish at infinity, we set $u(x, t)=\phi(\xi)=\hat{\phi}=\tilde{\phi}(\xi)$, where $\xi=x-v t$. Testing the singular behaviour of the solution $\left(\phi \sim \xi^{-p}\right)$ of the resulting ODE gives $p=2 / c$, suggesting the nonlinear transformation $\phi=\tilde{\phi}^{2 / c}$. Hence, after factoring out $(2 / c) \tilde{\phi}^{(2 / c-2)}$ we obtain

$$
v \tilde{\phi} \tilde{\phi}_{\xi}+\tilde{\phi} \tilde{\phi}_{\xi \xi}+\left(\frac{2}{c}-1\right) \tilde{\phi}_{\xi}^{2}+\frac{c}{2} \tilde{\phi}^{2}-\frac{c}{2} \tilde{\phi}^{4}=0
$$

which is completely of polynomial type in $\tilde{\phi}$ and its derivatives. Singular analysis of this equation indicates that $\tilde{\phi} \sim \xi^{-1}$, and for selected values of $c$ the equation passes the Painlevé test. Note that the transformation $\phi=\tilde{\phi}^{1 / c}$ would have worked equally well. The nonlinear term in the resulting ODE in $\tilde{\phi}$ would have been cubic rather than of fourth degree.

The above equation has no linear terms, hence we consider the lowest-degree terms. Upon substitution of $\tilde{\phi}=g=\exp (-K \xi)$ into the quadratic terms of $(58)$, we find

$$
f(K, v)=\frac{2}{c} K^{2}-v K+\frac{c}{2}=0
$$

We solve (59) for $v$, substitute that into (58) together with the expansion $\tilde{\phi}$

$$
\tilde{\phi}=\sum_{n=1}^{\infty} a_{n} g^{n}
$$

It turns out that an expansion in $g=\exp (-K \xi)$ itself works since (59) is quadratic, whereas in the preceding example $f(K, v)$ in (43) was cubic. This yields, upon equating the different powers of $g$, the recursion relation (with $n \geqslant 4$ )

$$
\begin{gathered}
\sum_{l=1}^{n-1}\left[K^{2} l^{2}-\left(\frac{2 K^{2}}{c}+\frac{c}{2}\right) l+\left(\frac{2}{c}-1\right) K^{2} l(n-l)+\frac{c}{2}\right] a_{n-l} a_{l} \\
-\frac{c}{2} \sum_{l=3}^{n-1} \sum_{m=2}^{l-1} \sum_{j=1}^{m-1} a_{n-1} a_{l-m} a_{m-j} a_{j}=0 .
\end{gathered}
$$


It is easy to determine that $a_{n}=A_{0}=$ constant. Using the formulae for sums of the powers of the first $n-1$ integers, we have after a rather tedious calculation

$$
\begin{aligned}
& A_{0}= \pm 1 \\
& K_{1}=-K_{2}=\frac{c}{\sqrt{2 c+4}}
\end{aligned}
$$

and using $K=K_{1}$ in (59) yields

$$
v=\frac{c+4}{\sqrt{2 c+4}}
$$

We restrict ourselves to waves travelling towards the right. The waves travelling to the left are obtained similarly with negative values for both $K$ and $v$.

To obtain the final solution we perform the following steps:

$$
\begin{aligned}
\tilde{\phi} & =\sum_{n=1}^{\infty} A_{0}\left(a_{0} g\right)^{n} \\
& = \pm F_{0}\left(a_{0} g\right) \\
& = \pm \frac{a_{0} g}{1-a_{0} g} \\
& =\mp \frac{\exp (-K \xi-\Delta)}{1+\exp (-K \xi-\Delta)} \\
& =\mp \frac{1}{2}\left[1-\tanh \left(\frac{K \xi+\Delta}{2}\right)\right]
\end{aligned}
$$

where we used (9) and (62) and where $a_{0}=-\exp (-\Delta)$. Finally, returning to the original variables,

$u(x, t)=\phi(x-v t)=\llbracket\left[\frac{1}{2}\left\{1-\tanh \left[\left(\frac{c}{2 \sqrt{2 c+4}}\right)\left(x-\frac{(c+4) t}{\sqrt{2 c+4}}\right)+\frac{\Delta}{2}\right]\right\}\right]^{2 / c}$.

This solution was obtained by Wang (1988) with a completely different method involving a clever trick. For $c=1$ it reduces to the well known solution of the Fisher equation (56) (Ablowitz and Zeppetella 1979, Wang 1988, Hereman and Angenent 1989)

$$
u(x, t)=\frac{1}{4}\left\{1-\tanh \left[\frac{1}{\sqrt{6}}\left(x-\frac{5 t}{\sqrt{6}}\right)+\frac{\Delta}{2}\right]\right\}^{2} .
$$

For $c=2$ we obtain

$$
u(x, t)= \pm \frac{1}{2}\left\{1-\tanh \left[\frac{1}{2 \sqrt{2}}\left(x-\frac{3 t}{\sqrt{2}}\right)+\frac{\Delta}{2}\right]\right\} .
$$

This result is a special case of that obtained from Painlevé analysis by Cariello and Tabor (1989) and Hereman (1990a).

\subsection{Special case}

To conclude let us briefly come back to the original Fisher equation (56)

$$
u_{t}-u_{x x}-u(1-u)=0
$$


for which the linear part admits the dispersion relation

$$
f(K, v)=K^{2}-v K+1=0 .
$$

Seeking a solution of

$$
\phi_{\xi \xi}+v \phi_{\xi}+\phi-\phi^{2}=0
$$

for $\phi$ as in $(60)$ but with $g=\exp [-(K / s) \xi]$, we readily obtain the recursion relation

$$
\left(\frac{n}{s}-1\right)\left(\frac{v K}{s}-\frac{n}{s}+1\right) a_{n}-\sum_{l=1}^{n-1} a_{l} a_{n-1}=0 .
$$

It is clear that $\tilde{K}=K / s \neq K_{1} \neq K_{2}$ implies $a_{1}=0$. Asking that $a_{2} \neq 0$ and $a_{3} \neq 0$ requires that $s=2$ and $s=3$. Hence, since $K_{1}=2 \tilde{K}$ and $K_{2}=3 \tilde{K}$ both solve $(70)$ one easily calculates $\tilde{K}=1 / \sqrt{6}$ and $v=5 / \sqrt{6}$. The coefficient of $a_{n}$ in the recursion relation, i.e.

$$
\tilde{K}^{2} n^{2}-v \tilde{K} n+1
$$

clearly factors into $\frac{1}{6}(n-2)(n-3)$. It is worthwhile mentioning that (70) only passes the Painlevé test provided $v^{2}=\frac{25}{6}$, for which (70) can be factored right away.

The above results could have been obtained by solving (72) with a solution of the form $a_{n}=A_{1} n+A_{0}$, in which case one would find that $a_{n}=\left(a_{0}\right)^{n}(n-1)$. The observant reader may wonder where this peculiar case with the special values for $K_{1}$ and $K_{2}$ is hidden in the above treatment of the gF equation. To see this, take $K_{1}$ and $v$ as in (63) and (64), and calculate the second solution of (59). This gives $K_{2}=c(c+2) /(2 \sqrt{2 c+4)}$. Observe that $K_{1}=p_{1} \tilde{K}$ and $K_{2}=p_{2} \tilde{K}$ with $p_{1}=2, p_{2}=c+2$ and $\tilde{K}=c /(2 \sqrt{2 c+4)}$. The factor $p_{2}$ is an integer iff $c$ is an integer. Setting $c=1$ gives $p_{1}=2, p_{2}=3$, and $\tilde{K}=$ $1 /\left(2 \sqrt{2 c+4)}\right.$. One thus retrieves the parameters $\tilde{K}, K_{1}, K_{2}$ and $v$ for the Fisher equation (56). The extra factor 2 now appearing in the denominators is due to the fact that $\phi=\tilde{\phi}^{2}$.

\section{Discussion and conclusion}

From the three examples, it should be clear that the direct method is now extended to cover analytical rather than rational expressions in real exponentials. These real exponentials satisfy the linearized equation or-if that is not present-the lowest-degree terms of the nonlinear equation. Although there may exist exponentials with different exponents, in most cases it suffices to consider one 'basic' exponential form. All the exponentials are powers of this common one. Physically, this means that we are working with commensurate frequencies.

The most crucial steps in the algorithm are now completely systematic. The needed nonlinear transformations follows from singular analysis (Painlevé analysis). The recursion relations are obtained in the desired form by using Cauchy's formula for products of series. The recursion relations can be solved for a polynomial-type solution by hand or with any symbolic program. The summation of the series expansions in closed form is now based on general, but simple formulae. All these steps are straightforward and do no longer need intuition, experience or hindsight.

In the near future we hope to treat equations (Hereman and Takaoka 1990), which do not have solutions in terms of hyperbolic functions. We also intend to look at the examples studied in this paper from the point of view of phase plane analysis. This will require a careful classification of the singular points of the linearized first-order systems, followed by an investigation of the dimension and stability of the manifolds 
through these singular (or critical) points. The theory for this goes back to theorems by Liapunov and Poincaré (Arnold 1983, ch 5, p 187). The final goal is then to find the closed-form representation corresponding to the homoclinic and heteroclinic orbits through the equilibrium points. Homoclinic trajectories in phase space will lead to pulse-type solutions such as $\operatorname{sech}^{n}$ ( $n$ integer); heteroclinic orbits correspond to kinktype solutions such as 1 -tanh. Jeffrey and Kakutani (1972) treated a combined KdV-Burgers equation along those lines. Recently, Coffey (1990b) carried out a phase plane analysis for (11) with $c=1$ and $c=2$, in a first attempt to justify the direct algebraic method as described and applied in his earlier paper (Coffey 1990a). Angenent (1988) calculated the speed of meromorphic travelling wave solutions for $u_{t}-u_{x x}=f(u)$, where $f(u)$ is of polynomial type with real coefficients and simple zero at $u=0$ and $u=1$, and for which $f^{\prime}(1)<0<f^{\prime}(0)$ holds. These conditions are satisfied for the Fisher equation (56). Phase plane analysis could lead to a better understanding of the applicability of the method to other equations. Also the connection between this method and its counterpart in the complex plane deserves further investigation. In particular, the use of Mittag-Lefler's theorem for the determination of the form of the solitary waves (Takaoka 1989).

Finally, we should mention that the method is readily applicable to $(2+1)$. dimensional equations such as the Kadomtsev-Petviashvili equation (Kadomtsev and Petviashvili 1970). Furthermore, the method allows one to solve coupled systems (Hereman 1988), discrete equations such as the Toda equation (Dash and Panigraphi 1990 ) and integral equations (Coffey 1990a). $N$ solitary wave solutions and implicit solutions can be obtained with a generalization of this method (see, e.g., Hereman et al 1986, Hereman 1988, 1990a, c and Banerjee et al 1990). The physical idea and the mathematical steps are pretty much the same but the calculations are much more involved.

\section{Acknowledgments}

One of the authors (WH) would like to acknowledge the support of the Air Force Office of Scientific Research under Grant No. 85-NM-0263. Professor Partha Banerjee (Department of Electrical Engineering, Syracuse University) and Professor Sigurd Angenent (Mathematics Department, University of Wisconsin-Madison) are thanked for various illuminating discussions. MT also acknowledges the support of the Research Institute for Mathematical Sciences of Kyoto University.

\section{References}

Ablowitz M and Zeppetella A 1979 Bull. Math. Biol. 41 835-40

Angenent S 1988 Meromorphic travelling waves Preprint

Arnold V I 1983 Geometrical Methods in the Theory of Ordinary Differential Equations (Berlin: Springer)

Banerjee P P, Daoud F and Hereman W 1990 A straightforward method for finding implicit solitary wave solutions of nonlinear evolution and wave equations J. Phys. A: Math. Gen. 23 521-36

Cariello F and Tabor M 1989 Physica D 39 77-94

Coffey M 1990a On series expansions giving closed form solutions of KdV-like equations SIAM J. Appl. Math. $\mathbf{5 0}$ in press

$1990 \mathrm{~b}$ Phase space analysis for the direct algebraic method for nonlinear evolution and wave equations SIAM J. Appl. Math. $\mathbf{5 0}$ in press

Conte R and Musette M 1989 J. Phys. A: Math. Gen. 22 169-77 
Dai X and Dai J 1989 Phys. Lett. 142A 367-70

Dash P C and Panigraphi M 1990 Construction of solitary wave solutions of the Toda lattice from real exponential solutions of the linear equation Phys. Lett. 80A to appear

Dey B $1986 J$ J. Phys. A: Math. Gen. 19 L9-12

Fokas A S and Ablowitz M J 1983 Nonlinear Phenomena (Lecture Notes in Physics 189) ed K B Wolf (Berlin: Springer) pp 137-83

Gardner C S, Greene J M, Kruskal M D and Miura R M 1967 Phys, Rev. Lett. 19 1095-7

Hereman W 1988 The construction of implicit and explicit solitary wave solutions of nonlinear partial differential equations Proc. Math. Soc. Egypt 57 in press

- 1990a Partially Integrable Evolution Equations in Physics, Proc. Summer School Theoretical Physics, Les Houches, France, March 21-28, 1990 ed R Conte and N Boccara (Dordrecht: Kluwer) pp 585-6

- 1990b Solitary wave solutions to coupled system of nonlinear evolution equations using MACSYMA Proc. IMACS 1st International Conference on Computational Physics, University of Colorado, Boulder, June 11-15, 1990 ed K Gustafson and W Wys (Boulder, CO: University of Colorado Press) pp 150-3

- 1990c Exact solitary wave solutions of nonlinear evolution and wave equations using MACSYMA Special Issue Comp. Phys. Commun. $\mathbf{4 2}$ in press

Hereman W and Angenent S 1989 MACSYMA Newsletter 6 11-8

Hereman W, Banerjee P P and Chatterjee M R 1989 J. Phys. A: Math. Gen. 22 241-55

Hereman W, Banerjee P P, Korpel A, Assanto G, Van Immerzeele A and Meerpoel A 1986 J. Phys. A: Math. Gen. 19 607-28

Hereman W, Korpel A and Banerjee P P 1985 Wave Motion 7 283-90

Hereman W and Takaoka M 1990 Exact solitary wave solutions of nonlinear PDE using MACSYMA, in preparation

Hereman W and Van den Bulck E 1988 Finite Dimensional Integrable Nonlinear Dynamical Systems ed P L G Leach and W.H Steeb (Singapore: World Scientific) 117-29

Hirota R 1980 Solitons (Topics in Current Physics 17) ed R K Bullough and P J Caudrey (Berlin: Springer) ch $5, \mathrm{pp}$ 157-75

Hooper A P and Grimshaw R 1988 Wave Motion 10 405-20

Hunter J K and Vanden-Broeck J-M 1983 J. Fluid Mech. 134 205-19

Ince E L 1956 Ordinary Differential Equations (New York: Dover)

Jeffrey A and Kakutani T 1972 SIAM Rev. 14 583-643

Kadomtsev B B and Petviashvili V I 1970 Sov. Phys.-Dokl. 15 539-41

Korpel A 1978 Phys. Lett. 68A 179-81

Rosales R R 1978 Stud. Appl. Math. 59 117-53

Schamel H 1973 J. Plasma Phys. 9 377-87

Spiegel M R 1968 Mathematical Handbook of Formulas and Tables (Shaum's Outline Series) (New York: McGraw-Hill)

Tagare S G and Chakrabarti A 1974 Phys. Fluids 17 1331-2

Takaoka M 1989 J. Phys. Soc. Japan 58 73-81 (addendum 3028)

Verheest F 1987 Proc. 1987 Int. Meeting on Plasma Physics, Kiev, April 6-12, 1987 Vol 2, ed A G Sitenko (Kiev: Naukovka Dumka) pp 115-8

Wadati M and Sawada K 1980 J. Phys. Soc. Japan 48 319-25

Wang X Y 1988 Phys. Lett. 131A 277-9

Wang X Y, Chu Z S and Lu Y K 1990 J. Phys. A: Math. Gen. 23 271-4

Weinstein M I 1986 Nonlinear Systems of Partial Differential Equations in Applied Mathematics (Lectures in Applied Mathematics 23) eds B Nicolaenko, D D Holm and J M Hyman (Providence, RI: American Mathematical Society) pp 23-30

Zabusky N J 1967 Nonlinear Partial Differential Equations ed W F Ames (New York: Academic) pp 223-58 